\title{
Valeur alimentaire du sérum de latex d'Hévéa
}

\author{
par R. FERRANDO et DANG QUAN DIEN
}

Le latex provient d'Hevea brasiliensts, arbre à caoutchouc, dont il constitue la sève. A côté de l'Hevea brasiliensis il convient également de mentionner Funtumia elastica d'Afrique, Castilloa elastica d'Amérique Centrale et Ficus elastica d'Asie. C'est l'Hevea brasiliensis qui est le plus répandu parmi les arbres à caoutchouc.

Fraîchement extrait de l'arbre, le latex contient 30 à 40 p. 100 de résidu sec et son $\mathrm{pH}$ est d'environ 6,2. Meyer (1) en a donné la composition suivante :

$$
\begin{aligned}
& \text { - eau ............6 } 60 \text { p. } 100 \\
& \text { - caoutchouc ......... } 32 \text { " } \\
& \text { - protéines ......... } 2 \text { " } \\
& \text { - lipides divers ....... } 2 \text { " } \\
& \text { - constituant inorganique } 4 \text { " }
\end{aligned}
$$

Ce liquide contiendrait également une quantité non négligeable d'antioxydants. Après concentration par centrifugation il reste un sérum dont la teneur en matière sèche est très variable (6 à 10 p. 100).

Des études ont été poursuivies par Archer et Selikar (2) puis Archer et Cockbain (3), sur les protéines du sérum de latex. Après congélation du sérum à $-25^{\circ} \mathrm{C}$., ces auteurs séparèrent les protides de ce sérum et les étudièrent par électrophorèse. Ils parvinrent ensuite (3) à isoler du sérum une $x$-globuline dont l'analyse élémentaire est la suivante :

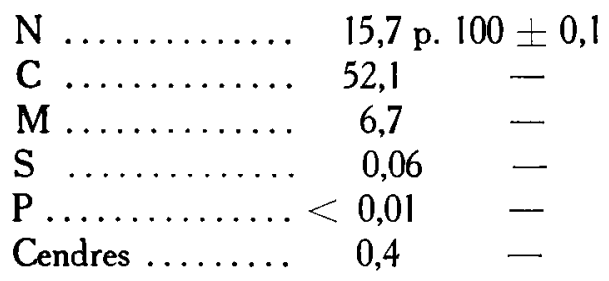

Cette $x$-globuline donne les réactions positives suivantes :

Biuret, Ninhydrine, Xanthoprotéique, Millon, Sakaguchi et Adamkiewicz.
L'odeur régnant dans une usine de préparation du caoutchouc, odcur voisine de celle du sérum de fromagerie émanant dans les fruitières, nous incita à étudier la valeur alimentaire du sérum de latex qui, dans les pays où se prépare la gomme, est rejeté à la rivière alors qüil pourrait peut-être servir à l'alimentation des animaux domestiques, du porc en particulier, dans des régions dont on connaît la grande pénurie des ressources alimentaires.

Les échantillons de sérum de latex que nous avons utilisés pour nos expériences contenaient de 0,109 à 0,612 g. p. 100 de matières protéiques.

Nous avons étudié la teneur en acides aminés libres du sérum grâce à la chromatographie sur papier.

Trois sérums ont été étudiés de la manière suivante $\left({ }^{*}\right)$ : dialyse prolongée contre de l'eau distillée. Les dialysats sont concentrés puis chromatographiés sur résine permutite $C^{50}$. L'élution se fait par l'ammoniaque $2 \mathrm{~N}$. Après élimination de l'ammoniaque on effectue la chromatographie bidimentionnelle sur papier Arches 301. Les solvants utilisés sont le phénol et la collidinebutidine. La mise en évidence des taches a été faite au moyen de la ninhydrine puis on a vaporisé sur les chromatogrammes une solution méthanolique de nitrate de cuivre pour assurer leur conservation.

Dans l'ensemble on trouve les mêmes taches dans chacun des chromatogrammes. Seule la proportion de la tache 1 (acide glutamique) varie en importance (cf. figure l).

On note également une faible tache 2 ayant un Rf. de 1 dans la phase phénol et de Rf 0 ou 0,1 dans la phase collidine. Seules, les taches 4 et 11 restent douteuses.

On a pu ainsi mettre en évidence l'acide glutamique, l'arginine, la lysine, l'alanine, la sérine,

(*) Par M. Rebeyrotte que nous tenons à remercier vivement. 


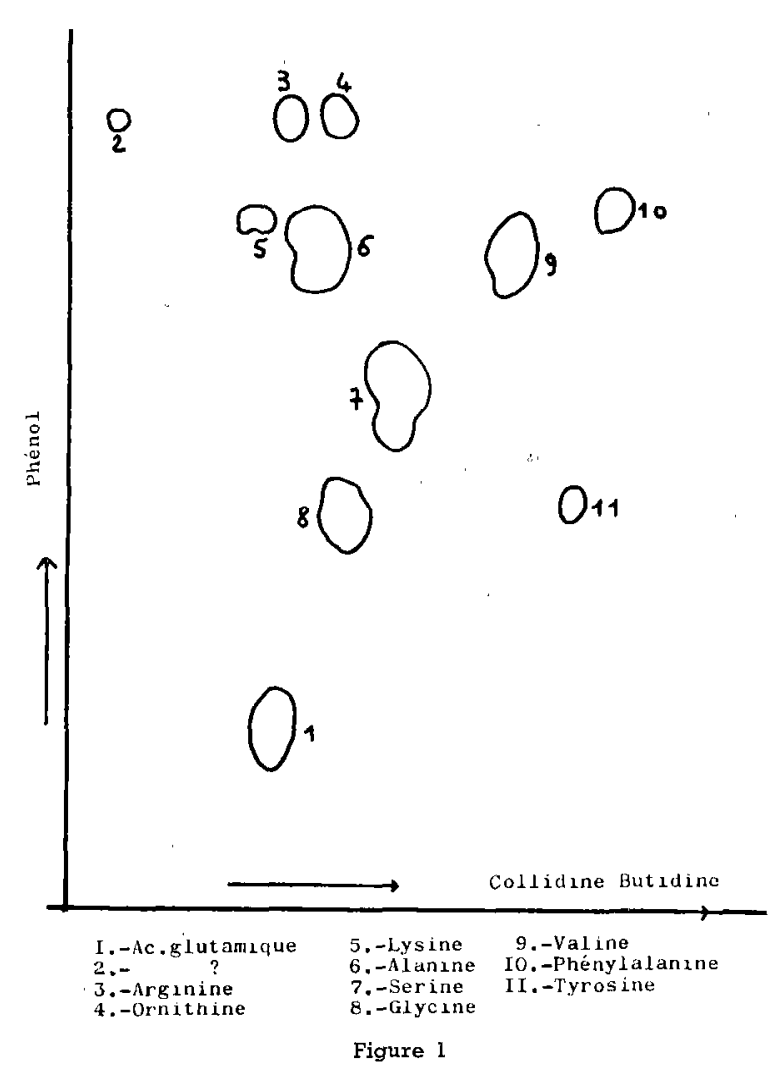

la glycine, la valine, la phénylalanine et probablement la tyrosine et l'ornithine.

L'efficacité protidique du sérum (4) a été examinée comparativement à celle du sérum de fromagerie préparé au Laboratoire.

Après une série d'essais poursuivis sur le rat blanc, nous avons pu constater l'absence de toxicité du produit. L'examen histologique des testicules de nos animaux d'expérience ne révèle pas d'altération de l'épithélium germinatif.

Nous avons alors déterminé dans une deuxième expérience l'efficacité Tprotidique du sérum de latex comparée à celle du sérum de fromagerie.

A cette fin, trois groupes, composés chacun de cinq rats pesant en moyenne $65,3 \mathrm{~g}$. reçurent un aliment de base composé de :

- Semoule de blé ........ 90 p. 100

- Huile d'arachide......... 5 -

- Mélange $\left({ }^{*}\right)$ et vitamines du complexe B ......... 5 -

Les vitamines $\mathrm{A}$ et $\mathrm{D}^{2}$ sont données sous forme de gouttes. Les trois groupes reçoivent au biberon et ad libitum les liquides suivants :

- Pour le groupe Témoin I, de l'eau

- Pour le groupe ...... II, du sérum de fromagerie

- Pour le groupe ....... III, du sérum de coagulation du latex.

Les quantités de liquide consommées sont mesurées quotidiennement. Les rats sont pesés également chaque jour.

Le tableau ci-dessous donne les résultats obtenus après 25 jours d'expérience.

On peut cependant considérer que la croissance excédentaire obtenue dans les groupes II et III est due, d'une part, à la quantité d'aliment de base ingéré en supplément aux protides absorbés avec la boisson. Cette quantité nulle pour le groupe I puisqu'il s'agissait d'eau se monte respectivement à 2,05 et à $0,93 \mathrm{~g}$. pour les groupes II et III absorbant le sérum de fromagerie et le sérum de latex. Pour ces protides excédentaires provenant de la boisson on peut considérer une efficacité protidique de 3,07 dans le cas du sérum de latex et de 5,09 dans le cas du sérum de fromagerie.

(*) R. Ferrando, Thèse, Doctorat ès-Sciences naturelles, 1952.

\begin{tabular}{|c|c|c|c|c|c|c|}
\hline Lots & $\begin{array}{l}\text { Supplément } \\
\text { au régime } \\
\text { de hage }\end{array}$ & $\begin{array}{c}\text { Gain Journalier } \\
\text { par rat } \\
(\text { E) }\end{array}$ & $\begin{array}{c}\text { Efficacite } \\
\text { protidique } \\
* *\end{array}$ & $\begin{array}{l}\text { Aliment de base } \\
\text { consomé } \\
\text { par jour }\end{array}$ & $\begin{array}{l}\text { Liquide } \\
\text { absorbé } \\
\text { par jour }\end{array}$ & $\begin{array}{l}\text { Poids des foies } \\
(\mathrm{g}) \text { en fin } \\
\text { d'cxpćrienoe }\end{array}$ \\
\hline I & - & 1,04 & 1,45 & 8,2 & 8,43 & 5 \\
\hline II & $\begin{array}{l}\text { Sérum de } \\
\quad \text { fromagerie }\end{array}$ & 2,14 & 2,28 & 11,5 & 9,91 & 6,81 \\
\hline III & $\begin{array}{l}\text { Sérum de } \\
\text { latex d'hévéa }\end{array}$ & 1,50 & 1,73 & 8,8 & 9,74 & \\
\hline
\end{tabular}

$\left.{ }^{* *}\right)$ Efficacité protidique $=\frac{\text { Gain de poids }(\mathrm{g})}{\text { quantité de protides ingérés }(\mathrm{g})}$ 
Il apparait ainsi qu'avec un régime de base uniquement composé de semoule de blé, le sérum de latex d'Hévéa permet un gain de croissance appréciable qui, sans atteindre celui obtenu avec le sénum de fromagerie, permet d'envisager une supplémentation des protides des céréales.

Des études de bilan et de digestibilité de la ration ont été aussi poursuivies sur des rats recevant le même régime que précédemment.

Chaque groupe comprend deux sujets. La boisson, distribuée à volonté, était mesurée chaque jour et comprenait soit de l'eau, soit du sérum de latex, soit du sérum de fromagerie dont la teneur en azote était ajustće par dilution à celle du sérum de latex. Après une période d'adaptation et de préparation de dix jours, l'expérience proprement dite dura une semaine. Le tableau suivant résume les résultats obtenus.
Il renfermait les vitamines suivantes :

— Riboflavine ........ 15,0 mg p. 100

- Pyridoxine ......... 7,8 -

- Thiamine ......... 7,5 -

- Acide nicotinique .... 30 -

- Pantothénate de Calcium 30

- Vitamine A .......... 400 U.I. p. 100

- Vitamine $\mathrm{D}_{2}$....... 400 U.I. -

Ce régime contenait $4,550 \mathrm{mg}$ d'azote par gramme soit 2,81 p. 100 de matières protéiques (cœef. 6,25). Un groupe A comprenant 5 rats, reçoit ce seul régime à volonté et comme boisson de l'eau. Un autre groupe B, également de 5 rats, reçoit ce même régime, sa boisson étant du sérum de latex contenant $0,175 \mathrm{mg}$. d'azote au gramme soit 0,109 p. 100 de matières protéiques, une des teneurs les plus faibles en protides que nous ayons enregistrée sur les échantillons de sérum de latex en notre possession.

Tableau II

\begin{tabular}{|c|c|c|c|c|c|}
\hline Croupes & $\begin{array}{l}\text { Supplément } \\
\text { au régime de } \\
\text { base }\end{array}$ & $\begin{array}{c}\text { Bilan azoté } \\
\text { (mg) }\end{array}$ & 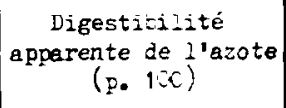 & $\begin{array}{c}\text { Gain } \\
\text { de poids moyen } \\
(\mathrm{g})\end{array}$ & $\begin{array}{l}\text { p. } 100 \text { d'azote fixé } \\
\text { en plus par rapport } \\
\text { aux témoins }\end{array}$ \\
\hline$I$ & 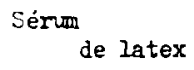 & +580 & 89 & 12 & 10 \\
\hline II & $\frac{\text { pation témoin }}{(\text { eau })}$ & $+528,6$ & 97 & 7,8 & Témoin \\
\hline III & $\begin{array}{l}\text { Sénum } \\
\text { de fromagerie }\end{array}$ & $+554,2$ & 92 & 10,12 & 4,9 \\
\hline IV & $\begin{array}{l}\text { Sérutio de latex } \\
\text { de }\end{array}$ & $+606,7$ & 92 & 12,5 & 14,9 \\
\hline
\end{tabular}

L'excès d'azote absorbé par rapport à l'aliment de base, seule nourriture du groupe II, a été de 12,3 p. 100 pour les rats du groupe I, de 12,5 p. 100 pour ceux du groupe III et de 12,4 p. 100 pour ceux du groupe IV.

Dans une troisième série d'expériences nous avons voulu examiner sur des rats pesant en moyenne 59 grammes quelles seraient les conséquences d'une supplémentation par le sérum de latex, d'un régime pauvre en protides.

Le régime de base utilisé était ainsi composé :

$\begin{array}{lll}\text { - Riz broyé .......... } & 45 & \text { p. } 100 \\ \text { - Fécule pomme de terre } & 47,5 & - \\ \text { - Huile d'arachide ..... } & 5 & - \\ \text { - Mélange minéral ..... } & 2,5 & -\end{array}$

Dans une première période les rats du groupe A perdent au total 2 grammes en 25 jours. Par rapport au poids initial la moyenne des pertes par pesée est de $-8,22$ grammes (au plus - 14; au moins - 1) Dans une deuxième période (18 jours) le gain est pour l'ensemble du groupe de 33 grammes, soit 1,5 gr jour/groupe.

Les sujets du groupe $B$ ne perdent pas de poids pendant la première période et gagnent même 6 grammes à la fin de cette période. Si l'on tient compte, comme pour les animaux de A, des fluctuations de poids par rapport au poids initial pendant les 25 premiers jours de l'expérience, la moyenne des pertes n'est que de $-3,7$ grammes (au plus $-8,5$; au moins $-0,5$ et un gain de +6 grammes lors de la dernière pesée). 
Dans la deuxième période de 18 jours le gain est pour l'ensemble du groupe de 47 grammes, soit 2,2 gr. jour/groupe.

\section{DISCUSSION ET CONCLUSIONS}

L'ensemble de ces analyses et de ces essais biologiques indique que le sérum de latex possède une valeur alimentaire assez comparable à celle du sérum de fromagerie. Les protides du sérum de latex doivent ainsi pouvoir servir à supplémenter ceux du riz et d'autres céréales. $\mathrm{Au}$ cours de nos expériences nous avons été gêné par la faible teneur en protéines du sérum de latex. Il nous était difficile d'en faire absorber aux rats plus de 10 à $20 \mathrm{~cm}^{3}$ par jour, soit environ un apport de matières protéiques de 10 à $25 \mathrm{mg}$. au moins et de 60 à $120 \mathrm{mg}$. au plus. Dans l'alimentation du porc les quantités distribuées doivent être plus importantes et de l'ordre de 5 litres au début de la croissance pour atteindre 18 à 20 litres en fin d'engraissement. De telles quantités représentent donc pour les plus faibles teneurs de $5-10$ gr et jusqu'à $20-40$ gr. de matières protéiques par jour. Pour les sérums plus riches les chiffres sont respectivement de 30 et de 120 grammes par jour.

Un tel apport n'est pas négligeable dans les pays où la pénurie d'aliments du bétail est pour ainsi dire chronique. Une étude s'impose en vue d'une meilleure récupération des sérums de latex. Elle devra être complétée par une expéri- mentation sur le porc que l'un d'entre nous se propose de poursuivre dans le cadre du VietNam. Ce n'est qu'une fois cette expérimentation terminée que nous pourrons conclure définitivement sur la valeur alimentaire du sérum de latex d'Hévéa. Si cette valeur alimentaire élail confirmée, l'élevage du porc dans les Pays d'Extrême-Orient, d'Afrique et d'Amérique du Sud pourrait en recevoir une impulsion. De graves problèmes d'alimentation humaine risqueraient alors d'être plus aisément résolus.

Laboratoire d'Alimentation de l'Ecole Nationale Vétérinaire d'Alfort et Laboratoire Central de Recherches Vétérinaires d'Alfort.

\section{BIBLIOGRAPHIE}

1. Meyer (K.-H.). - Natural and Synthetic High Polymers. Vol. IV, 1942, p. 173, NewYork. Interscience, publish.

2. Archer (B.-L.) et Sekhar (B.-C.). - Biochem. Journ., 1955, 61, 504-8.

3. Archer (B.-L.) et Cockbain (E.-G.). - Biochem. Journ., 1955, 61, 508-12.

4. Ferrando (R.) et Dang Quan Dien. - C. $R$. Acad. Sci., 1957, 245, 860-1.

\section{SUMMARY}

\section{Nutritive value of Hevea Latex Sera.}

The nutritional value of Hevea Latex Sera was studied both chemically and biologically. The percentage of protides ranged from 0.109 to $0.612 \mathrm{gm}$ per cent, and the following amino-acids were detected : glutamic acid, arginine, lysine, alanine, serine, glycine, valine, phenylalanine, and probably tyrosine and ornithine.

The nutritional value of the sera was compared to that of dairy-cheese extracts in feeding trials on rats and found to be comparable.

The authors consider that Hevea may be useful as a source of proteins in a country where the food is generally insufficient for livestock.

\section{RESUMEN}

\section{Valor alimenticio del suero de latex de Hevea.}

En un conjunto de analisis y de ensayos biológicos los autores buscan conocer el valor alimenticio del suero de latex. Estudiando los prótidos encuentran que su cantidad varía según las muestras de 0,109 a $0,612 \mathrm{~g}$ por $100 \mathrm{y}$ ponen en evidencia los acidos aminados siguientes : acido glutámico, arginina, lisina, alinina, serina, glicina, valina, fenilalanina y probablemente tirosina y ornitina. Enseguida comparan el valor de éste suero con el de la fabricación de queso, en la alimentatión de ratas y encuentran que los valores alimenticios del uno y del otro son bastante comparables.

Piensan que éste aporte protídico podria ser interesante en aquellos paises donde se explotan los heveas y donde la alimentatión del ganado es generalmente insuficiente. 